\title{
Water Surfaces in the Oil Fields
}

\author{
BY MARCEL R. DALY, SEATTLW, WASH.
}

(New York Meeting, February, 1918)

(A contribution to the study of the conditions of equilibrium of the "free surface" of a water body inclosed in a porous medium.)

IN a recent paper on Geologic Structure in the Cushing Oil and Gas Field, Oklahoma, ${ }^{1}$ Carl H. Beal has pointed out some interesting peculiarities in the distribution of the hydrocarbons and the disposition of the "planes of separation" between the oil and the water (water surfaces). Mr. Beal's principal statements on this matter may be summarized as follows:

I. In every important dome in the Cushing field, which has not been complicated by folding along its sides, the oil area in each of the three oil and gas sands studied (Layton, Wheeler and Bartlesville) apparently extends farther down on the long axis of the dome than it does on the steeper sides. The result is that water is encountered at a lesser depth below datum plane (sea level) on the steep sides of the dome than along the ends where the beds are more gently tilted.

II. In the Layton and Wheeler sands (to which the detailed study of water surfaces has been almost entirely confined), the water surface is not level, but dips at a gentle angle (maximum dip $100 \mathrm{ft}$. to the mile) away from the center of the structure. This inclination existed prior to development, and it has been rendered greater by the rapid extraction of oil and gas.

To these statements, the following observations are added: The dominant structural feature in the Cushing field is a broad north and south anticlinal fold, along whose axis the domes are distributed (Fig. 1, p. 897), and the more important oil areas in each sand lie on the west side of the field. In many cases, gas has been forced to the east side of the structure, sometimes as far as the water line.

From the distribution of the oil and gas bodies, Mr. Beal concludes that the hydrocarbons in this region probably migrated from the west or the northwest.

It seems as if these different features would be susceptible of some 
mechanical interpretation, and the writer wishes to develop here briefly his views on the subject. ${ }^{2}$

At first, we may dispose of a possible objection. . The propriety of likening the flow of underground waters to the flow of surface waters through pipes or channels, has been sometimes questioned. It is obvious that most of the formulas, and especially the "coefficients" used in practical hydraulic engineering for free-water flow, could hardly be expected to apply to a liquid flowing through a porous medium. Such formulas are merely a theoretical framework on which are hung the result of experiment, and whenever the conditions of experiment become different, the formulas may only yield misleading results. On the other hand, it cannot be denied that a surface flow and an underground flow are phenomena of the same order. As Slichter remarks, " "the water moves in the underground current for the same reason that water moves in the surface streams," and the general law of motion, in both cases, is grounded on the same fundamental principles. Bernouilli's Theorem, upon which hinges the whole fabric of hydromechanics, is simply an application of such principles; and wherever the assumptions on which this theorem is further based can be admitted (steady and non-sinuous flow of a perfect fluid), the theorem itself may be applied. In most cases, conditions would permit such an application to underground flow as well as to surface flow, within sufficient limits of approximation, and the close analogy of both groups of phenomena becomes apparent.

A second observation bears on the nature of the surface of equilibrium of a liquid, whether it be a free liquid or a liquid inclosed in a porous medium.

Whenever a free liquid is at rest and its free surface (or the surface which is in contact with a gas or vapor) is submitted to uniform pressure -for instance, atmospheric pressure-this surface is horizontal. And the same is true of a liquid inclosed in a porous medium, provided we should consider as "free surface" the upper surface of the saturated zone. In both cases the free surface is an equipotential surface and its form is determined by the condition that at every point it must be normal to the lines along which the material particles of water tend to travel following the direction of the most rapid potential fall (lines of force). Here, this direction is vertical, the only force acting on the particle being the force of gravity; hence the equipotential surface is horizontal.

There may be instances, such as illustrated in Fig. 1, in which the

${ }^{2}$ At the time this paper is written (October 1917), Bulletin No. 658 has not yet been issued by the U. S. Geological Survey, and Mr. Beal's abstract is the only source of information available to the writer in regard to the special features presented by the water surfaces in the Cushing field. Therefore, the conclusions presented here are subject to revision, in so far as they would conflict with some fact unknown.

${ }^{3}$ C. S. Slichter: The Motions of Underground Waters. U. S. Geological Survey Water Supply Paper No. 67 (1902), 17. 
pressure above the liquid would vary from one point to another. If the pressure in $A$ be the atmospheric pressure, for instance, and the pressure in $B$ be greater than atmospheric, the free surface in both parts of the container would no more be on the same level; $c d$, in $B$, would be lower than $a b$, in $A$. Conditions may be imagined in nature where the same result would obtain. Such would be the case in a flexed sand stratum (Fig. 2), where the gas pressure may be greater in $B$ than in $A$. But in both instances, the surfaces $a b$ and $c d$, considered individually, would be horizontal. The horizontality of the free surface of a liquid in contact with a gas or vapor, whether it be a free liquid above ground or an underground liquid inclosed in a porous medium, becomes thus a direct consequence of the state of rest; it is the expression of static equilibrium.

Conditions are entirely different when the liquid, whether free or not, is in a state of motion. The free surface of a body of water in steady motion, which is the condition most generally to be considered, will be normal at any point to the resultant of (1) the force required to reduce a particle of water at that point to rest or to uniform motion in a straight

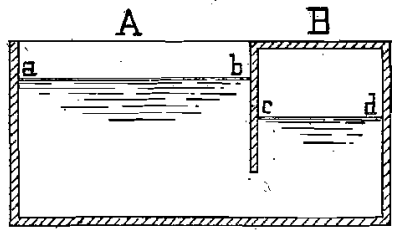

FIg. 1.

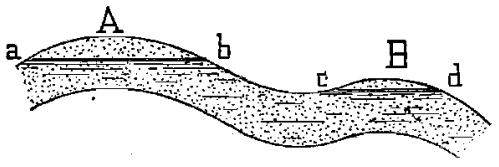

FIG, 2.

line, and (2) the force of gravity on the particle. The first force would be inclined, ${ }^{4}$ whereas the second is vertical. Hence, the resultant would be inclined also, and so would the free surface at that point. The differential equation of the longitudinal profile of such a surface shows it to be a curved surface, which becomes a plane when the flow is uniform. The fact that the body of water is inclosed in a porous medium would introduce an additional set of conditions, due to the resistance against motion of this intervening medium, but the resulting "free-surface" would still remain a curved surface and the same conclusions would obtain. Thus, the characteristic of the free surface of a liquid in motion, whether free or inclosed in a porous medium, is its non-horizontality, or its continuous slope. ${ }^{5}$

The peculiarities recorded by $\mathrm{Mr}$. Beal in the Cushing field, and heretofore summarized, imply the existence of gradually sloping water surfaces in open channels or incompletely filled conduits; and such conditions

${ }^{4}$ Unless the direction of the motion should be vertical, which is a case limit and is not to be considered here.

5 The presence of a sheet of oil resting on the surface of the water would not change the scope of these conclusions. 
would, in their turn, imply the motion of the underlying water bodies. The conclusion is that, in the Cushing field, the "edge waters" on which the oil and gas rest are presumably in a state of motion, and the following observations are offered as a consequence of this preliminary remark.

The phenomenon of "backwater" is well known in hydraulics. It occurs when an open channel is obstructed by a dam or weir. If the water is flowing in the channel with uniform motion previous to the construction of the weir, the raising of the weir will cause the water to

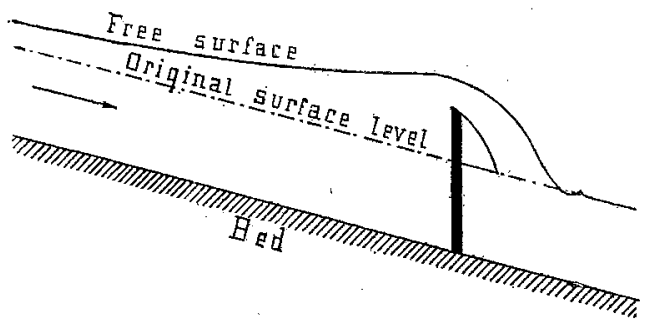

FIG. 3.

back upstream, increasing the depth in the direction of flow (Fig. 3). The same phenomenon may be observed, on a reduced scale, in a shallow stream, where the bed is strewn with cobbles or boulders. Whenever the water encounters an emergent boulder, it rises against its upstream face, and, dependent on conditions, it may either flow over it or simply turn around it. If the boulder is not submerged (Fig. 4), as between boulders, and downstream from them, the stream maintains its normal depth, the line of intersection $a b c d e$ of the obstacle with the water surface

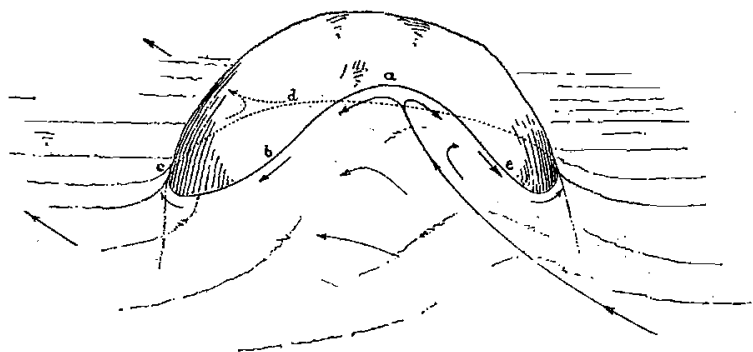

FIG. 4. - (The arrows marls the direction of the current.)

in front of it decreases in height on both sides of the stone, its highest point $a$ being roughly located under the crest of the latter; and the water surface is seen to dip away from the center of the stone, especially in the upstream direction and on both flanks. Where the rush of the water is sufficient and the dimensions of the boulder are not too large, the obstacle may be entirely submerged. In this case, provided the amount of submergence keeps under certain limits, the water surface above the stone is again seen to dip in every direction a'way from the crest.

In both preceding instances (dam and boulder) the channel is sup- 
posed to dip in the direction of the current, and the motion of the water is due to gravity. But there are cases in nature where such conditions are reversed; i.e., where the current runs up dip and against gravity. For instance, one may have observed the waves breaking on a gently rising sand beach. Whenever the wave collapses, the waters are thrown forward and dash, for the most part, upshore. They form a sheet that spreads ahead and laterally. If these ascending waters encounter a shingle or a large pebble, they will rise against the obstacle, exactly as in the two previous instances, and the same deformation obtains for the free surface of the liquid. The rule is this a general one, as far as observation goes; and it is further confirmed by mathematical analysis.

Let us turn now to the Cushing field and consider, for instance, the sketch map showing the generalized structure of the Layton sand. ${ }^{6}$ If the oil and gas bodies have migrated from the west or northwest, as Mr. Beal admits, then the anticlinal fold, which cuts the field with a north-south trend and along whose axis the domes are distributed, must have acted as an obstacle in the path of the transporting waters flowing through the sand from this same western or northwestern direction. The sand stratum through which this flow has taken place would have to be considered, from the standpoint of hydraulics, as the equivalent of an "open channel" or an "incompletely filled pipe," at least in the neighborhood of the field. This is evidenced by the disposition of the water surfaces themselves, on which the oil and gas rest, and the variableness of their slope, that has been rendered greater by extraction since development has begun. Thus, the conditions would be similar to those which would be met if a dam, provided with a series of contracted weirs, were erected across a wide and shallow river, or if a line of boulders were deposited in the bed of a shallow stream, athwart its course. The series of peculiarities noted by Mr. Beal would be the natural consequence of such a disposition. The water would have to rise to a higher level against the steeper flanks of the domes than against their lower extremities or at points where the stream is permitted to cross the structure through "cols" or depressions. In consequence, water would be found at a lesser depth, under datum plane, on the steeper sides of the domes than farther down along their axis. Further, the water surfaces would have to be inclined away from the center of the anticlinal folds, exactly as they are in the case represented in Fig. 4., and for the same reasons.

The increase in slope of the water surfaces that has been noted after the rapid extraction of gas and oil in the field, would have to be traced to the decrease of the counter pressure, resulting in a suction and a consequent increase in the velocity of the water current. The slope of the free surface of a water body in the state of motion is a function of its velocity. An increase in the velocity of the current would necessarily fol- 
low the rapid decrease of counter-pressure resulting from the escape of the gas, or the reduction in volume of the fluids that rest on the water itself.

The velocities in such a body of moving waters would not be the same everywhere. The velocity would reach a maximum wherever the current passes freely across the folds; and would reach a minimum where the edges of the stream abut on the protruding parts of the structure, such as the domes, against which the current would be deflected, or at points where the structure of the ground would induce the formation of local eddies. Such places would be the most favorable ones for the accumulation of the transported materials, oil and gas, and these primary deposits would, for the most part, face an upstream direction; i.e., they would mostly be found on the western side of the anticlinal fold. Moreover, when laid down, these transported materials would settle according to density. The oil would largely remain where first deposited, whereas gas would diffuse through the sand. It would tend to reach the higher places (the crest of the folds, the summit of the domes, etc.) but, at the same time, it would invade those parts of the sand stratum that are free of water and where oil has no access, especially on the reverse side (eastern side) of the structure, facing the downstream direction. Gas may then accumulate in such places as far as the water line.

A question may be raised here. How is it possible that the transported materials, especially the oil which remains in contact with the upper surface of the water, would reach a state of rest when the underlying waters are still in a state of motion and remain so permanently? This may be readily answered. The writer has already pointed out in a preceding paper ${ }^{7}$ that for each insoluble material transported by a current flowing through a porous medium, whether this material be heavier or lighter than the transporting liquid, there is a certain velocity limit under which the transportation ceases to be possible and deposition ensues. This result is essentially due to the difference of density between the two materials, the transporting and the transported, and to the resistance against movement of the porous medium. The resulting action may be directed either down or up, depending upon whether the transported material is heavier or lighter than the transporting liquid. Now, the velocity of an underground flow is generally very low when the origin of the movement is simply due to a static head, as would be the case today, in the present instance, and this velocity would easily fall under the velocity limit corresponding to oil and gas deposition; so that oil would be at rest upon the moving sheet of water simply for the reason that the motion of the latter would have reached a stage at which it can no more transport the oil, exactly as a sluggish stream flows over the gravels of its bed which it cannot "scour" any longer. This remark leads us to a further conclusion. It is obvious that if the 
present velocity of underground flow does not permit any more oil transportation, conditions must have been somewhat different in the past, when the hydrocarbons were being collected and accumulated. For reasons given elsewhere, ${ }^{8}$ the writer considers it very doubtful that the pressure due to a static head alone could ever, under normal conditions, be adequate to produce the velocity required for transportation. We are thus brought to consider the intervention of some other force, whose action would be dynamic rather than static, and the probability of some intimate connection between petroliferous accumulation and diastrophic deformation becomes once more apparent.

\section{Conclusions}

I. In the Cushing field, the "edge waters" on which the oil and gas rest are presumably in a state of motion; the main direction of flow proceeding from the west or northwest.

II. The peculiarities pointed out by Mr. Beal—such as: the variation in the level of the water surfaces, which are found higher along the steep flanks of the domes and further down on their long axes; the slope of the water surfaces; away from the axis of the structure; the localization of the more important oil areas on the west side of the folds; the concentration of some gas bodies on the eastern side, as far as the water line, etc.would be as many expressions of such a state of motion.

III. The disposition of the water surfaces noted in the Cushing field may be encountered elsewhere, but there is no apparent reason why this disposition should be considered as a general rule. These forms depend on a state of movement which may not always exist, although such a condition is probably more frequent than is generally admitted. On the other hand, the peculiarities themselves bear an intimate relation to each other; they are parts of a system, and wherever one of them is noted, the others are likely to be found. This consideration may have some practical importance.

IV. The water surfaces would not be planes, but surfaces with a double curvature, as the motion would be non-uniform.

$V$. The increase in the slope of the water surfaces resulting from the rapid extraction of oil and gas could not be expected to remain permanent. Eventually, this slope would have to be reduced in proportion as the extraction itself declines. But the general advance of the water bodies would probably be final.

VI. The facts observed and the conclusions heretofore set forth, suggest, by way of inference, that, in the past, the cause of motion cannot be ascribed to static pressure alone, as the resulting velocity of flow would not have been sufficient to promote the concentration of the hydrocarbons. The writer is once more of the opinion that the real source of this motion is to be found in diastrophic deformation. 